\title{
The Association between Glomerular Diameter and Secondary Focal Segmental Glomerulosclerosis in Chronic Kidney Disease
}

\author{
Ryo Zamamia,b Kentaro Kohaguraa, ${ }^{a}$ Kojiro Kinjyo ${ }^{a, b}$ Takuto Nakamura ${ }^{a}$ \\ Takanori Kinjo $^{a}$ Masanobu Yamazato $^{a}$ Akio Ishida ${ }^{a}$ Yusuke Ohya ${ }^{a}$ \\ aDepartment of Cardiovascular Medicine, Nephrology and Neurology, Graduate School of Medicine, University of \\ the Ryukyus, Okinawa, Japan; 'Dialysis Unit, University of the Ryukyus Hospital, Okinawa, Japan
}

\section{Keywords}

Glomerular hypertrophy · Secondary focal segmental glomerulosclerosis · Chronic kidney disease

\begin{abstract}
Introduction: When nephron loss occurs, the glomerular filtration rate (GFR) is suggested to be maintained by glomerular hypertrophy, but excessive hypertrophy can rather lead to the formation of focal segmental glomerulosclerosis (FSGS), thereby causing progressive kidney damage. However, it is not clear how much glomerular hypertrophy leads to the formation of FSGS. We examined the association between glomerular diameter and FSGS lesions in chronic kidney disease (CKD) patients. Methods: We recruited 77 patients who underwent renal biopsy during 2016-2017; however, those identified with primary FSGS and glomerulonephritis with active glomerular lesion were excluded. We evaluated the maximal glomerular diameter (Max GD), an indicator of glomerular size, in each renal biopsy specimen and examined its association with FSGS lesion. Results: The median age, blood pressure, and estimated GFR of the patients were 53 years, 122/70 $\mathrm{mm} \mathrm{Hg}$, and 65 $\mathrm{mL} / \mathrm{min} / 1.73 \mathrm{~m}^{2}$, respectively. The optimal cutoff threshold of Max GD for predicting the presence of FSGS lesions, assessed by receiver operating characteristic curve analysis, was determined to be at $224 \mu \mathrm{m}$ (area under the curve, 0.81;
\end{abstract}

sensitivity, 81\%; specificity, 72\%). Multivariate logistic regression analyses demonstrated that Max GD $\geq 224 \mu \mathrm{m}$ was significantly associated with the presence of FSGS lesions, independent of other confounding factors (odds ratio, 11.70; 95\% confidence interval, 1.93-70.84). Discussion/Conclusion: Glomerular hypertrophy (Max GD $\geq 224 \mu \mathrm{m}$ ) has been associated with FSGS lesions in CKD patients and may reflect the limits of the compensatory process.

(c) 2021 The Author(s).

Published by S. Karger AG, Basel

\section{Introduction}

Chronic kidney disease (CKD) can cause a variety of disorders. In patients with CKD, renal dysfunction may progress even when the activity of the underlying disease might seem well controlled. As a secondary factor associated with the progression of such renal dysfunction, studies have reported the importance of glomerular hypertrophy attributed to glomerular hypertension [1]. With the decrease in the nephron number, the remaining nephrons are compensatory enlarged, and the total glomerular filtration rate (GFR) is then maintained by an increase in the single nephron GFR in patients with CKD [2-4]. In addition, a study on renal transplant donors reported that long-term renal outcome was comparable to that of gen- karger@karger.com www.karger.com/kbr

Karger $\stackrel{\text { ' }}{5}$

GOPEN ACCESS
(C) 2021 The Author(s)

Published by S. Karger AG, Basel

This is an Open Access article licensed under the Creative Commons Attribution-NonCommercial-4.0 International License (CC BY-NC) (http://www.karger.com/Services/OpenAccessLicense), applicable to the online version of the article only. Usage and distribution for commercial purposes requires written permission.
Correspondence to:

Kentaro Kohagura, kohagura@med.u-ryukyu.ac.jp 
eral controls despite the decrease in the nephron number by approximately $50 \%$ [5]. Renal transplantation donors have demonstrated parallel increases in single kidney cortical volume and GFR without glomerular hypertension [6]. However, new-onset hypertension, diabetes mellitus (DM), and obesity, which could cause glomerular hypertension, have been often associated with an increased risk of end-stage renal disease in living renal transplant donors $[7,8]$. In rats, severe renal mass reduction $(>50 \%)$ was found to result in progressive glomerular injury due to glomerular hypertrophy along with glomerular hypertension $[3,9]$. These findings suggested that the progression of renal dysfunction after nephron loss depends on the presence of glomerular hypertension.

A certain condition such as DM and protein overload can cause dilatation of afferent arterioles, which leads to glomerular hypertrophy associated with an increased intraglomerular pressure $[10,11]$. The increased mechanical strain on podocytes due to glomerular hypertrophy induces podocyte detachment from glomerular capillaries, which can ultimately lead to the formation of focal segmental glomerulosclerosis (FSGS) lesions [2]. The decreased glomerular filtration area due to FSGS lesions is seen to further increase glomerular hypertension to residual nephrons, resulting in a vicious cycle. A previous report suggested that hyperperfusion injury occurs in a variety of glomerulonephritis and diabetic kidney disease cases with varying frequencies [12]. Therefore, renal dysfunction can progress independent of the underlying disease activity.

Based on these findings, we hypothesized that single nephron GFR is increased by glomerular hypertrophy without glomerular injury in a compensatory range, whereas FSGS lesions due to glomerular hypertrophy might be formed beyond the compensatory range. However, there have been few reports examining the threshold for the morbid glomerular hypertrophy. Therefore, in this study, we have investigated the specific glomerular size that is associated with FSGS lesions, a potential marker of the limits of the compensatory process, using renal biopsy specimens.

\section{Materials and Methods}

\section{Study Participants}

In this cross-sectional observational study, we consecutively examined 133 patients with CKD who underwent renal biopsy because of renal function impairment or urinalysis abnormalities at the University Hospital of Ryukyus between January 1, 2016, and December 31,2017. We defined primary FSGS as patients who met

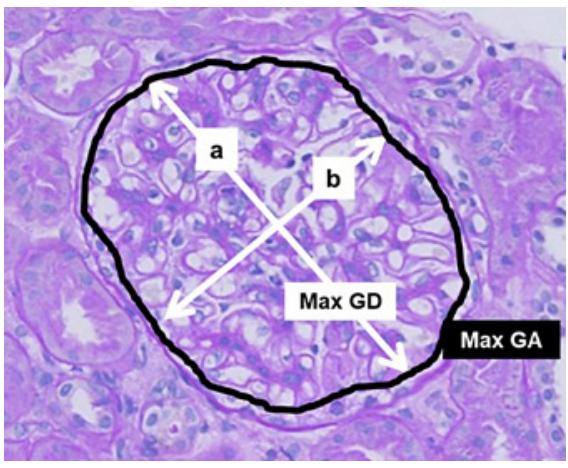

Fig. 1. Representative microphotographs of a glomerulus for the measurement of Max GD and Max GA. Max GD; maximal glomerular diameter; Max GA, maximal glomerular area.

the criteria for nephrotic syndrome, had no evidence of other renal disease except for FSGS lesions in pathological findings, and had diffuse foot process effacement on electron microscopy [13]. We excluded patients with primary FSGS $(n=4)$ from the study. Because FSGS lesions also result from postinflammatory scarring of glomerulonephritis with marked inflammatory response [14], patients with necrotizing crescentic glomerulonephritis $(n=29)$ were also excluded. In addition, patients with endocapillary proliferative glomerulonephritis $(n=10)$, which reportedly can cause glomerular enlargement by itself, were excluded [15]. To minimize sampling bias, patients with $<5$ glomeruli in renal biopsy specimens $(n=13)$ were also excluded. Finally, a total of 77 patients were enrolled in this study (see online suppl. Fig. 1; for all online suppl. material, see www.karger.com/doi/10.1159/000515528). We did not exclude the patients with nephrotic syndrome because it has little effect on association between glomerular size and FSGS lesions. The study protocol was approved by the Institutional Review Board of the University of Ryukyus (\#879) and was performed in accordance with the Declaration of Helsinki. All the participants of this study provided written informed consent.

\section{Pathological Analysis}

All kidney specimens were obtained by percutaneous needle biopsy. The percentage of global sclerotic glomeruli among all glomeruli (\%GSG) was evaluated as a pathological indicator of glomerular injury. We defined the extent of FSGS lesions as the percentage of glomeruli with FSGS lesions among the nonsclerotic glomeruli. Glomerular density was defined as the number of nonsclerotic glomeruli per unit area of cortex $\left(\mathrm{mm}^{2}\right)[16,17]$. The extent of interstitial fibrosis was semiquantitatively scored according to the percentage of fibrotic lesions in the entire cortical area. Arteriolosclerosis has been defined as the presence of arteriolar hyalinosis in each renal specimen.

The glomerular diameter was calculated as the mean value of the maximal diameter and the maximal chord perpendicular to it of each glomerulus. In a previous study, it was reported that the glomerular diameter measured using the same method in the present study highly correlated with glomerular area $(r=0.98)$ [18]. The glomerular area was defined as the area described by the outer capillary loops of each glomerulus. We also defined the glomer- 
ular diameter and the area of the most enlarged glomerulus in each kidney biopsy specimen as the maximal glomerular diameter (Max GD) and the maximal glomerular area (Max GA), respectively (shown in Fig. 1) [18]. Furthermore, we calculated the glomerular volume $(\mathrm{GV})$ from the measured GA using the Weibel and Gomez method as follows: $\mathrm{GV}=(\mathrm{GA})^{3 / 2} \times \beta / d$, where $\beta$ is a dimensionless shape coefficient ( $\beta=1.38$ for spheres) and $d$ is a size distribution coefficient that is used to adjust for variations in glomerular size $[19,20]$. The analysis used $d=1.01$ as done in previous studies [21, 22]. We defined the GV of the most enlarged glomerulus in each kidney biopsy specimen as the maximal GV (Max GV). In this study, we used the Max GD rather than the mean glomerular diameter in evaluating the glomerular size of each patient. There could be a wide variety of glomerular sizes, such as hypertrophied and collapsed glomeruli even in the same kidney tissue. Therefore, using the mean glomerular diameter may not reflect the glomerular hypertrophy derived from glomerular hypertension. In addition, because not all glomeruli were cut at the center, the sampling bias was expected to be larger when the mean glomerular diameter was used. Therefore, we examined an association between Max GD and FSGS lesions.

According to the Columbia classification, the presence of FSGS lesions has been defined as one or more glomeruli with segmental increase in the matrix obliterating the capillary lumina in each specimen [14]. The glomerular diameter and the presence of FSGS lesions were separately measured using different investigators. Both investigators were blinded to patient information. Images were then analyzed using the ImageJ software.

\section{Data Collection}

Hypertension was defined as a systolic blood pressure (SBP) of $\geq 140 \mathrm{~mm} \mathrm{Hg}$ and/or a diastolic blood pressure (DBP) of $\geq 90 \mathrm{~mm}$ $\mathrm{Hg}$ and/or the use of antihypertensive medication. The body mass index (BMI) was calculated as body weight/height squared $(\mathrm{kg} /$ $\mathrm{m}^{2}$ ). Fasting blood samples were used in measuring the serum total cholesterol, high-density lipoprotein and low-density lipoprotein cholesterol, triglyceride, uric acid, creatinine, hemoglobin $A_{1 c}$ $\left(\mathrm{HbA}_{1 \mathrm{c}}\right)$, and glucose levels. Urinary protein and creatinine levels were measured using first spot morning urine samples. Proteinuria was evaluated using the urinary protein-to-creatinine ratio (with both protein and creatinine levels measured in grams). DM was defined as a fasting plasma glucose level of $\geq 7.0 \mathrm{mmol} / \mathrm{L}$, an HbA1c level of $\geq 6.5 \%$, and/or the use of any medication for diabetes. Dyslipidemia was defined as a serum low-density lipoprotein cholesterol level of at least $3.60 \mathrm{mmol} / \mathrm{L}$, a triglyceride level of at least $1.69 \mathrm{mmol} / \mathrm{L}$, a high-density lipoprotein cholesterol level of $<1.04 \mathrm{mmol} / \mathrm{L}$, and/or the use of medications for dyslipidemia. The estimated GFR (eGFR) was calculated using the Japanese equation [23]. Smoking history was determined through an interview.

\section{Statistical Analysis}

All values were expressed as medians (interquartile ranges) and percentages for continuous variables and for categorical variables, respectively. Clinical characteristics were compared using the Mann-Whitney $U$ test for continuous variables and the $\chi^{2}$ test for categorical variables. Spearman's rank correlation was used to evaluate the association between 2 continuous variables. Two-sided tests with significance levels of $p$ values $<0.05$ were used. The receiver operating characteristic curve was plotted to determine the optimal cutoff value of Max GD for predicting FSGS lesions, and the area under the curve was calculated. Multivariate logistic regression analysis was also performed to identify the independent factors associated with the presence of FSGS lesions after adjusting for age, sex, BMI, SBP, DM, eGFR, use of renin-angiotensin-aldosterone system inhibitor (RAASi), and Max GD (continuous variables and dichotomized variables by cutoff value) or Max GV, which has been reported to be associated with glomerular hypertrophy and glomerular hypertension [10, 24-26]. A similar multivariate logistic regression analysis was also conducted by adding $\%$ GSG as an explanatory variable instead of eGFR. In addition, we performed a multiple regression analysis to investigate factors associated with the extent of FSGS lesions after adjusting for age, sex, BMI, SBP, DM, eGFR, use of RAASi, and Max GD (dichotomized variables by cutoff value). Multivariate logistic regression analysis was also performed using the same explanatory variables to identify factors associated with the presence of FGSG lesions only in the patients with IgA nephropathy or nonnephrotic syndrome. We further examine an association of the cutoff value of Max GD with changes in eGFR among those with IgA nephropathy. Data were analyzed using the JMP 12 software (SAS Institute Inc., Cary, NC, USA).

\section{Results}

\section{Distribution of the Primary Renal Diagnosis}

Based on renal biopsy findings, the following primary renal diagnoses were made in 77 patients: IgA nephropathy $(n=45)$, membranous nephropathy $(n=10)$, interstitial nephritis $(n=4)$, minimal change disease $(n=4)$, nephrosclerosis $(n=3)$, thin basement membrane disease $(n=2)$, IgA vasculitis $(n=2)$, diabetic nephropathy $(n=$ $2)$, and other miscellaneous diseases $(n=5)$. We compared the primary renal diagnosis according to the presence of FSGS lesions. The results showed that the prevalence of IgA nephropathy, the most common type of primary renal diagnosis, was not significantly different between the patients with and without FSGS lesions (57 vs. $63 \%, p=0.47)$. The patients with minimal change disease, which was difficult to completely distinguish from primary FSGS, were distributed only in the non-FSGS group (shown in online suppl. Table 1).

\section{Relationship between Max GD and FSGS Lesions}

The median age of all patients was 53 years (range: 35-64 years), and $56 \%$ of them were males. The median eGFR was $65 \mathrm{~mL} / \mathrm{min} / 1.73 \mathrm{~m}^{2}$ (range: $51-88 \mathrm{~mL} / \mathrm{min} / 1.73$ $\mathrm{m}^{2}$ ), and the median BMI was $25 \mathrm{~kg} / \mathrm{m}^{2}$ (range: $22-28 \mathrm{~kg} /$ $\mathrm{m}^{2}$ ). None of the patients had low birth weight. The median Max GD was $210 \mu \mathrm{m}$ (interquartile range: 197-237 $\mu \mathrm{m})$ (online suppl. Fig. 2). The cutoff value that predicted the presence of FSGS lesions was $224 \mu \mathrm{m}$ for Max GD
Glomerular Diameter and Secondary

Focal Segmental Glomerulosclerosis
Kidney Blood Press Res 2021;46:433-440 DOI: $10.1159 / 000515528$ 
Table 1. Clinical characteristics by Max GD cutoff value $(224 \mu \mathrm{m})$

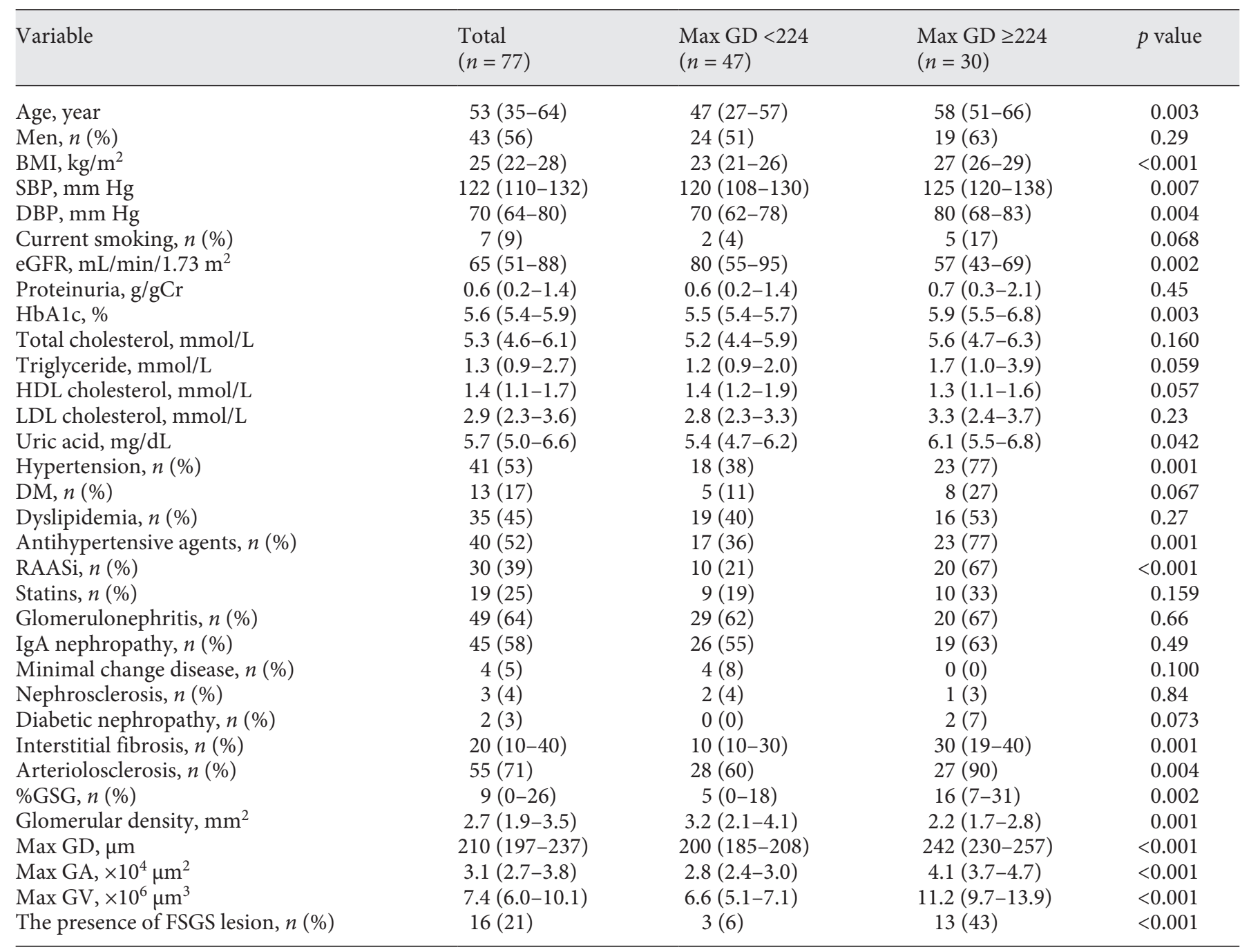

Data are presented as median with the interquartile range or number (\%). \%GSG, the percentage of global sclerotic glomeruli among all glomeruli; BMI, body mass index; DBP, diastolic blood pressure; eGFR, estimated glomerular filtration rate; FSGS, focal segmental glomerulosclerosis; GD, glomerular diameter; GS, global sclerosis; RAASi, renin-angiotensin-aldosterone system inhibitor; SBP, systolic blood pressure; Max GD; maximal glomerular diameter; Max GA, maximal glomerular area; DM, diabetes mellitus; Max GV, maximal glomerular volume; HDL, high-density lipoprotein; LDL, low-density lipoprotein; $\mathrm{Hb}_{1 \mathrm{c}}$, hemoglobin $\mathrm{A}_{1 \mathrm{c}}$.

(area under the curve, 0.81 ; sensitivity, $81 \%$; and specificity, 72\%). Compared with lower Max GD $(<224 \mu \mathrm{m})$, those with higher Max GD $(\geq 224 \mu \mathrm{m})$ were older, and had, higher BMI, SBP, DBP, HbA1c, uric acid, the extent of interstitial fibrosis, \%GSG, prevalence of hypertension, use of antihypertensive agents and use of RAASi, arteriolosclerosis, and lower eGFR (Table 1). The prevalence of patients with FSGS lesions was $21 \%$. Compared with patients without FSGS lesions, those with FSGS lesions had higher values for BMI, prevalence of nephrosclerosis, ex- tent of interstitial fibrosis, \%GSG, Max GD, Max GA, and Max GV (shown in online suppl. Table 2; Fig. 2). In univariate analysis, there was a significant correlation between the extent of FSGS lesions and Max GD ( $\rho=0.43$, $p<0.001$ ). We measured glomerular density but could not estimate the total number of nephrons because we did not record the renal total cortical volume. Since glomerular density alone has also been reported to be associated with glomerular size and renal prognosis [17], so we examined the relationship between glomerular density and 


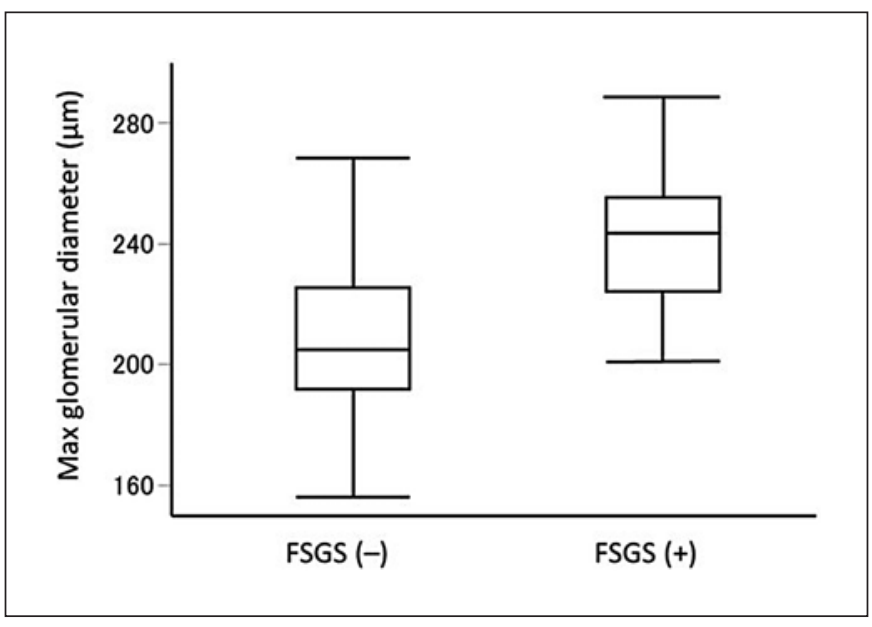

Fig. 2. Relationship between the presence of FSGS lesions and Max GD. FSGS, focal segmental glomerulosclerosis; Max GD; maximal glomerular diameter.

clinical parameters. The results showed that glomerular density was positively correlated with eGFR $(\rho=0.59, p<$ $0.001)$ and inversely correlated with Max GD $(\rho=-0.47$, $p<0.001)$, but was not associated with the presence of FSGS lesions $(p=0.083)$. Proteinuria was seen to be strongly affected by the underlying disease as we have included renal biopsy cases in this study. When nephrotic syndrome was excluded ( $n=63$ ), we founded a positive correlation between Max GD and proteinuria ( $\rho=0.30, p$ $=0.019$ ). Multivariate logistic regression analysis demonstrated that Max GD (continuous variable) was significantly associated with the presence of FSGS lesions, even after adjusting for potential confounding factors (Table 2, Model 1). In this study, Max GD was highly correlated with Max GA $(\rho=0.96, p<0.001)$ or Max GV $(\rho=0.96$, $p<0.001)$. When Max GV was included as a variable instead of Max GD in the multivariate analysis, Max GV remained as a significant factor related to the presence of FSGS lesions (Table 2, Model 2). When the Max GD (dichotomized variables by cutoff value) was used as an explanatory variable, higher Max GD was founded to be significantly associated with the presence of FSGS lesions (Table 2, Model 3). When \%GSG was added as an explanatory variable instead of eGFR, both higher Max GD and $\%$ GSG were found to be significantly associated with FSGS lesions (Table 2, Model 4). In addition, we also performed a multiple regression analysis to identify factors associated with the extent of FSGS lesions. As a result, higher Max GD was significantly associated with the extent of FSGS lesions, independent of other confounding factors $(\beta=0.419, p=0.003)$. Since this study included a variety of renal disease, we examined the association between FSGS lesions and glomerular size only in the group of IgA nephropathy, which had the largest number of patients. As a result, we founded a significant association between higher Max GD and the presence of FSGS lesions independent for other confounders (odds ratio, 15.25; 95\% confidence interval, 1.02-228.65). When multivariate analysis was performed only in the patients without nephrotic syndrome $(n=63)$, Max GD $\geq 224 \mu \mathrm{m}$ was associated with the presence of FSGS lesions, independently of other confounders (odds ratio, 34.78; 95\% confidence interval, 3.26-370.71).

\section{Changes in eGFR according to the Presence of}

Glomerular Hypertrophy Defined as $\geq 224 \mu \mathrm{m}$ of Max GD

In this study, we investigated the relationship between glomerular hypertrophy and renal outcome. Since primary renal disease could affect renal outcome, we examined changes in eGFR among limited patients with IgA nephropathy $(n=45)$. In the median follow-up of 986 days (interquartile range: 507-1,309 days), there was no significant difference in slope of eGFR among those with and without glomerular hypertrophy defined as Max GD $>224 \mu \mathrm{m}\left(-5.5\right.$ vs. $\left.-4.5 \mathrm{~mL} / \mathrm{min} / 1.73 \mathrm{~m}^{2}, p=0.70\right)$. However, in the nonnephrotic syndrome group $(n=44)$, the percentage of RAASi users were significantly higher in the group with Max GD $\geq 224 \mu \mathrm{m}$ (66.7 vs. $19.2 \%$, $p<$ 0.001 ), although there was no significant difference in proteinuria (median 0.6 vs. $0.5 \mathrm{~g} / \mathrm{gCr}, p=0.20$ ).

\section{Discussion/Conclusion}

This study has demonstrated a significant association between FSGS lesions and higher Max GD $(\geq 224 \mu \mathrm{m}$, cutoff value), independent of confounding factors in patients with CKD; this suggests the existence of a threshold of 224 $\mu \mathrm{m}$ for Max GD associated with FSGS lesions. In addition, higher Max GD was also significantly associated with the extent of FSGS lesions. These findings suggest that glomerular hypertrophy exceeding $224 \mu \mathrm{m}$ for Max GD is associated with glomerular structural injury.

In this study, we used 3 methods to measure glomerular size: diameter, area, and volume. Max GD was highly correlated with Max GA and Max GV $(\rho=0.96, p<0.001$ for both), and glomerular size by any measurement was significantly associated with the presence of FSGS lesions in multivariate logistic regression analysis. Glomerular 
Table 2. OR for the presence of FSGS lesions

\begin{tabular}{|c|c|c|c|c|c|}
\hline Age, year & $1.02(0.99-1.06)$ & $1.03(0.97-1.10)$ & $1.03(0.97-1.11)$ & $1.03(0.97-1.10)$ & $0.96(0.89-1.03)$ \\
\hline Men, $n(\%)$ & $1.02(0.34-3.19)$ & $1.26(0.28-6.04)$ & $1.24(0.27-5.65)$ & $0.87(0.20-3.76)$ & $0.82(0.15-4.35)$ \\
\hline $\mathrm{BMI}, \mathrm{kg} / \mathrm{m}^{2}$ & $1.28(1.10-1.52)^{*}$ & $1.19(0.98-1.48)$ & $1.20(0.98-1.47)$ & $1.20(0.97-1.48)$ & $1.20(0.96-1.50)$ \\
\hline RAASi user & $2.45(0.80-7.76)$ & $1.01(0.21-4.88)$ & $1.07(0.23-5.06)$ & $0.66(0.14-3.18)$ & $0.31(0.05-2.02)$ \\
\hline $\mathrm{eGFR}, \mathrm{mL} / \mathrm{min} / 1.73 \mathrm{~m}^{2}$ & $0.99(0.97-1.01)$ & $1.04(0.99-1.10)$ & $1.05(0.99-1.10)$ & $1.04(0.99-1.09)$ & \\
\hline \%GSG (\%) (per SD increase) & $2.60(1.50-4.86)^{*}$ & & & & $4.32(1.56-12.02)^{*}$ \\
\hline Max GD, $\mu \mathrm{m}$ (per $10 \mu \mathrm{m}$ increase) & $1.45(1.18-1.86)^{*}$ & $1.48(1.12-2.09)^{*}$ & & & \\
\hline Max GV, $\mu \mathrm{m}^{3}$ (per $10^{6} \mu \mathrm{m}^{3}$ increase) & $1.03(1.01-1.05)^{*}$ & & $1.03(1.01-1.05)^{*}$ & & \\
\hline Max GD $\geq 224 \mu \mathrm{m}$ & $11.22(2.83-44.34)^{*}$ & & & $11.70(1.93-70.84)^{*}$ & $10.59(1.82-61.69)^{*}$ \\
\hline
\end{tabular}

area and volume are difficult to measure in daily practice, whereas glomerular diameter is easy to measure. Therefore, we selected Max GD as the main outcome.

Bhathena [27] have investigated the association between glomerular size and the presence of FSGS lesions in renal biopsy specimens of patients with oligomeganephronia, unilateral agenesis, and unilateral nephrectomy. They have demonstrated that the group with FSGS lesions had a larger glomerular size than the group without FSGS lesions. On the other hand, no significant difference was determined in glomerular size between the groups with FSGS lesions, irrespective of the underlying disease. Based on these findings, it was concluded that there is a threshold for glomerular hypertrophy that cannot be increased further, and when it was exceeded, it resulted in the formation of FSGS lesions. In this present study, the prevalence of FSGS lesions significantly increased with a Max GD $\geq 224 \mu \mathrm{m}$, thus supporting their conclusion.

Although FSGS lesions are caused by a variety of etiologies, including circulating permeability factors, genetic defects, viruses, drugs, and adaptation to elevated glomerular capillary pressures and flow rates (adaptive FSGS), a common condition among them is podocyte depletion [13]. Brenner and colleagues [3] have demonstrated that glomerular hypertension, glomerular hyperfiltration, and glomerular hypertrophy contribute to adaptive FSGS. Based on the findings of ultrastructural studies, Kriz and Lemley [28]. reported that as the glomerular tuft enlarges, the resulting mechanical forces on podocytes promote hypertrophy of the podocyte cell bodies. They speculated the existence of a maximum cytoplasmic mass of podocyte and an upper limit of the adaptability of the foot process in response to glomerular hypertrophic growth When glomerular hypertrophy occurs beyond that limit, podocyte hypertrophy will eventually reach a breaking point, resulting in focal foot process detachment and podocyte loss [29]. As podocytes are terminally differentiated and incapable of self-renewal, a critical reduction in podocyte number will result in FSGS lesions [13]. In the present study, the prevalence of FSGS lesions was seen to increase with a Max GD of $224 \mu \mathrm{m}$ as the threshold value. This result suggested that a Max GD of $224 \mu \mathrm{m}$ can be the threshold at which podocyte hypertrophy reaches the upper limit and causes podocyte detachment.

A previous report suggested that hyperperfusion injury characterized by glomerulomegaly and FSGS lesions occurs in a variety of glomerulonephritis and diabetic kidney disease cases with varying frequencies [12]. A large-scale Japanese cohort study demonstrated that both glomerulomegaly and FSGS lesions were associated with an increased risk for composite renal events such as dialysis and doubling of a serum creatinine level among biopsy-proven diabetic nephropathy [30]. One of the pathological features of the Oxford classification that helps in predicting the risk for the progression of IgA nephropathy includes segmental glomerulosclerosis; it was also demonstrated to be an independent predictive factor of end-stage renal disease [31]. Kataoka et al. [18] have ex- 
amined the association between Max GD and renal outcome in IgA nephropathy and reported that glomerular hypertrophy with a Max GD $>242.3 \mu \mathrm{m}$ was associated with poor renal prognosis, defined as a 1.5-fold increase in serum creatinine value. They also reported that the ability to predict renal outcome based on the Oxford score for IgA nephropathy was significantly improved by adding the Max GD of $\geq 245.9 \mu \mathrm{m}$ [32]. Furthermore, they concluded that a pathological threshold existed for glomerular size, discriminating between morbid glomerular and physiological glomerular hypertrophy. Although, we did not find significant difference in changes of eGFR between the patients with and without glomerular hypertrophy defined as Max GD of $\geq 224 \mu \mathrm{m}$ among those with IgA nephropathy, the patients with glomerular hypertrophy had a significantly higher rate of RAASi users to achieve similar levels of proteinuria comparing to those without it. These findings may indirectly suggest that Max GD $\geq 224 \mu \mathrm{m}$ would be associated with worse renal outcome if the aggressive therapy with RAASi was not applied.

Another previous report has shown that increased GV was directly linked to hypertension and preceded rather than compensated for glomerulosclerosis [33]. Integrating the results of the present study and previous findings, it can be suggested that glomerular hypertrophy exceeding a Max GD of $224 \mu \mathrm{m}$ has a prognostic value for renal outcome.

This study has several potential limitations. As we have used needle biopsy specimens, we could not avoid sampling bias. In particular, FSGS lesions may be false negative due to focal distribution. Therefore, patients with $<5$ nonsclerotic glomeruli were excluded, but the sampling bias could still not be completely excluded. Our study included various underlying diseases. Especially in glomerulonephritis, FSGS lesions may be formed as scars due to inflammation rather than hemodynamics [14]. However, when we performed multivariate logistic regression analysis only in the group of IgA nephropathy, we founded a significant association between Max GD $\geq 224 \mu \mathrm{m}$ and the presence of FSGS lesions. This result might suggest that FSGS lesions were associated with the hemodynamic mechanism even in glomerulonephritis. Further studies on patients without renal disease, such as those in autopsy, are required to elucidate the exact threshold of Max GD that associates with the appearance of FSGS lesions. As the present investigation was a cross-sectional observational study, we were unable to demonstrate a causal relationship. Moreover, we could not measure the intraglomerular pressure due to the difficulty in its measure- ment in humans. Therefore, it remains unclear whether the presence of a morbid threshold is due to glomerular hypertrophy itself or due to an increased intraglomerular pressure. As shown in Table 1, patients with higher Max GD $(\geq 224 \mu \mathrm{m})$ had higher BMI, SBP, DBP, HbA1c, prevalence of hypertension, use of antihypertensive agents and use of RAASi, and lower eGFR compared to those with lower Max GD $(<224 \mu \mathrm{m})$. Obesity, DM, systemic hypertension, increased RAAS, and decreased nephron number were reportedly associated with glomerular hypertension [34]. Therefore, these results supported that glomerular hypertrophy indirectly reflects the presence of intraglomerular high blood pressure. Finally, the results of this study may not be applicable to all CKD patients as we have only recruited patients who underwent renal biopsy.

In conclusion, a potential threshold might exist for glomerular hypertrophy (Max GD >224 $\mu \mathrm{m}$ ), which can be associated with FSGS lesions in patients with CKD. The presence of such a threshold may reflect the limits of the compensatory process and the link to progressive deterioration of renal function. Further studies are warranted to investigate its predictive value for renal outcome.

\section{Acknowledgments}

We are grateful to Mr. Morihiro Ota and Mr. Masato Nohara for their skillful technical assistance in histological preparation and staining. The authors would like to thank Enago (www.enago. jp) for English language review.

\section{Statement of Ethics}

The study protocol was approved by the Institutional Review Board of the University of Ryukyus (\#879) and was performed in accordance with the Declaration of Helsinki. All the participants of this study provided written informed consent.

\section{Conflict of Interest Statement}

Dr. Kohagura has received speaker's fees from Nippon Boehringer Ingelheim Co., Ltd., Mitsubishi Tanabe Pharma Co., Ltd., Astellas Pharma Inc., MSD K.K., Ono Pharmaceutical Co., Ltd., AstraZeneca K.K., and Taisho Toyama Pharmaceutical Co., Ltd. Dr. Ohya has received speaker's fees from Nippon Boehringer Ingelheim Co., Ltd., Takeda Pharmaceutical Co., Ltd., and Daiichi Sankyo Pharmaceutical Co., Ltd. Dr. Zamami, Dr. Kinjyo, Dr. Nakamura, Dr. Kinjo, Dr. Yamazato, and Dr. Ishida have no conflicts of interest to disclose. 


\section{Funding Sources}

This study was not funded by any grant.

\section{Author Contributions}

R.Z. and K. Kohagura conceived of the presented idea. R.Z. developed the theory and performed the computations. K. Kinjyo, T.N., and T.K. verified the analytical methods. K. Kohagura, M.Y., A.I., and Y.O. supervised the findings of this work. All authors discussed the results and contributed to the final manuscript.

\section{References}

1 Jacobson HR. Chronic renal failure: pathophysiology. Lancet. 1991 Aug;338(8764): 419-23.

2 Fries JW, Sandstrom DJ, Meyer TW, Rennke HG. Glomerular hypertrophy and epithelial cell injury modulate progressive glomerulosclerosis in the rat. Lab Invest. $1989 \mathrm{Feb} ; 60(2)$ : 205-18.

3 Hostetter TH, Olson JL, Rennke HG, Venkatachalam MA, Brenner BM. Hyperfiltration in remnant nephrons: a potentially adverse response to renal ablation. Am J Physiol. 1981 Jul;241(1):F85-93.

4 Denic A, Mathew J, Lerman LO, Lieske JC, Larson JJ, Alexander MP, et al. Single-nephron glomerular filtration rate in healthy adults. N Engl J Med. 2017 Jun;376(24):234957.

5 Fehrman-Ekholm I, Elinder CG, Stenbeck M, Tydén G, Groth CG. Kidney donors live longer. Transplantation. 1997 Oct;64(7):976-8.

6 Lenihan CR, Busque S, Derby G, Blouch K, Myers BD, Tan JC. Longitudinal study of living kidney donor glomerular dynamics after nephrectomy. J Clin Invest. 2015 Mar;125(3): $1311-8$.

7 Matas AJ, Berglund DM, Vock DM, Ibrahim HN. Causes and timing of end-stage renal disease after living kidney donation. Am J Transplant. 2018 May; 18(5):1140-50.

8 Grams ME, Sang Y, Levey AS, Matsushita K, Ballew S, Chang AR, et al. Kidney-failure risk projection for the living kidney-donor candidate. N Engl J Med. 2016 Feb;374(5):411-21.

9 Bidani AK, Mitchell KD, Schwartz MM, Navar LG, Lewis EJ. Absence of glomerular injury or nephron loss in a normotensive rat remnant kidney model. Kidney Int. 1990 Jul; 38(1):28-38.

10 Tonneijck L, Muskiet MH, Smits MM, Van Bommel EJ, Heerspink HJ, Van Raalte DH, et al. Glomerular hyperfiltration in diabetes: mechanisms, clinical significance, and treatment. J Am Soc Nephrol. 2017 Apr;28(4): 1023-39.

11 Zeller K, Whittaker E, Sullivan L, Raskin P, Jacobson HR, Hughes V, et al. Effect of restricting dietary protein on the progression of renal failure in patients with insulin-dependent diabetes mellitus. N Engl J Med. 1991 Jan;324(2):78-84.

12 Bohle A, Biwer E, Christensen JA. Hyperperfusion injury of the human kidney in different glomerular diseases. Am J Nephrol. 1988; $8(3): 179-86$.
13 De Vriese AS, Sethi S, Nath KA, Glassock RJ, Fervenza FC. Differentiating primary, genetic, and secondary FSGS in adults: a clinicopathologic approach. J Am Soc Nephrol. 2018 Mar;29(3):759-74.

14 D’Agati VD, Fogo AB, Bruijn JA, Jennette JC. Pathologic classification of focal segmental glomerulosclerosis: a working proposal. Am J Kidney Dis. 2004 Feb;43(2):368-82.

15 Pound SE, MacDonald MK, Thomson D. Diffuse proliferative glomerulonephritis: how many types? Histopathology. 1987 Mar;11(3): $227-43$.

16 Viggiano D, Nigro M, Sessa F, Vignolini G, Campi R, Serni S, et al. The number of nephrons in different glomerular diseases. PeerJ. 2019 Sep 4;7:e7640.

17 Tsuboi N, Kawamura T, Koike K, Okonogi H, Hirano K, Hamaguchi A, et al. Glomerular density in renal biopsy specimens predicts the long-term prognosis of IgA nephropathy. Clin J Am Soc Nephrol. 2010 Jan;5(1):39-44.

18 Kataoka H, Ohara M, Honda K, Mochizuki T, Nitta K. Maximal glomerular diameter as a 10-year prognostic indicator for IgA nephropathy. Nephrol Dial Transplant. 2011 Dec;26(12):3937-43.

19 Weibel ER. Stereological methods. Practical methods for biological morphometry. London: Academic Press; 1979. Vol. 1; p. 44-5, 131-4.

20 Samuel T, Hoy WE, Douglas-Denton R, Hughson MD, Bertram JF. Applicability of the glomerular size distribution coefficient in assessing human glomerular volume: the Weibel and Gomez method revisited. J Anat. 2007 May;210(5):578-82.

21 Fulladosa X, Moreso F, Narváez JA, Grinyó JM, Serón D, Sero'n D, et al. Estimation of total glomerular number in stable renal transplants. J Am Soc Nephrol. 2003;14(10):26628.

22 Hughson MD, Samuel T, WE H, JF B. Glomerular volume and clinicopathologic features related to disease severity in renal biopsies of African Americans and Whites in the Southeastern United States. Arch Pathol Lab Med. 2007;131:1665-72.

23 Matsuo S, Imai E, Horio M, Yasuda Y, Tomita K, Nitta K, et al. Revised equations for estimated GFR from serum creatinine in Japan. Am J Kidney Dis. 2009 Jun;53(6):98292.

24 Hoy WE, Bertram JF, Denton RD, Zimanyi M, Samuel T, Hughson MD. Nephron num- ber, glomerular volume, renal disease and hypertension. Curr Opin Nephrol Hypertens. 2008 May;17(3):258-65.

25 Hoy WE, Hughson MD, Zimanyi M, Samuel T, Douglas-Denton R, Holden L, et al. Distribution of volumes of individual glomeruli in kidneys at autopsy: association with age, nephron number, birth weight and body mass index. Clin Nephrol. 2010 Nov;74(Suppl 1): 105-12.

26 Denic A, Mathew J, Nagineni VV, Thompson RH, Leibovich BC, Lerman LO, et al. Clinical and pathology findings associate consistently with larger glomerular volume. J Am Soc Nephrol. 2018 Jul;29(7):1960-9.

27 Bhathena DB. Focal glomerulosclerosis and maximal glomerular hypertrophy in human nephronopenia. J Am Soc Nephrol. 1996 Dec: 7(12):2600-3.

28 Kriz W, Lemley KV. A potential role for mechanical forces in the detachment of podocytes and the progression of CKD. J Am Soc Nephrol. 2015 Feb;26(2):258-69.

29 D'Agati VD. Podocyte growing pains in adaptive FSGS. J Am Soc Nephrol. 2017 Oct; 28(10):2825-7.

30 Furuichi K, Shimizu M, Yuzawa Y, Hara A, Toyama T, Kitamura H, et al. Clinicopathological analysis of biopsy-proven diabetic nephropathy based on the Japanese classification of diabetic nephropathy. Clin Exp Nephrol. 2018 Jun;22(3):570-82.

31 Shi SF, Wang SX, Jiang L, Lv JC, Liu LJ, Chen $\mathrm{YQ}$, et al. Pathologic predictors of renal outcome and therapeutic efficacy in IgA nephropathy: validation of the Oxford classification. Clin J Am Soc Nephrol. 2011 Sep;6(9): 2175-84.

32 Kataoka H, Moriyama T, Manabe S, Kawachi $\mathrm{K}$, Ushio Y, Watanabe S, et al. Maximum glomerular diameter and oxford MEST-C score in IgA nephropathy: the significance of timeSeries changes in pseudo- $\mathrm{R} 2$ values in relation to renal outcomes. J Clin Med. 2019 Dec; 8(12):2105.

33 Hughson MD, Puelles VG, Hoy WE, Douglas-Denton RN, Mott SA, Bertram JF. Hypertension, glomerular hypertrophy and nephrosclerosis: the effect of race. Nephrol Dial Transplant. 2014 Jul;29(7):1399-409.

34 Helal I, Fick-Brosnahan GM, Reed-Gitomer B, Schrier RW. Glomerular hyperfiltration: definitions, mechanisms and clinical implications. Nat Rev Nephrol. 2012 Feb;8(5):293300 . 